\title{
Magnetotransport of electrically induced two-dimensional hole gases in undoped GaSb quantum wells
}

\author{
Kenji Shibata $\odot,{ }^{1,2, *}$ Matija Karalic, ${ }^{2}$ Christopher Mittag, ${ }^{2}$ Thomas Tschirky, ${ }^{2}$ Christian Reichl, ${ }^{2}$ Hiromu Ito, ${ }^{1}$ \\ Katsushi Hashimoto, ${ }^{3,4}$ Toru Tomimatsu, ${ }^{3}$ Yoshiro Hirayama ${ }^{1},{ }^{3,4,5}$ Werner Wegscheider, ${ }^{2}$ Thomas Ihn, ${ }^{2}$ and Klaus Ensslin $\circledast^{2}$ \\ ${ }^{1}$ Tohoku Institute of Technology, Sendai 982-8577, Japan \\ ${ }^{2}$ Solid State Physics Laboratory, ETH Zurich, 8093 Zurich, Switzerland \\ ${ }^{3}$ Graduate School of Science, Tohoku University, Sendai 980-8578, Japan \\ ${ }^{4}$ Center for Spintronics Research Network, Tohoku University, Sendai 980-8577, Japan \\ ${ }^{5}$ Center for Science and Innovation in Spintronics, Tohoku University, Sendai 980-8577, Japan
}

(Received 18 June 2020; accepted 20 August 2020; published 9 September 2020)

\begin{abstract}
We have performed magnetotransport measurements on electrically induced two-dimensional hole gases in undoped $\mathrm{GaSb} / \mathrm{AlSb}$ quantum wells. The mobilities of the holes are sufficient to observe Shubnikov-de Haas oscillations for a few teslas of perpendicular magnetic field. We extracted the effective masses of holes in the valence bands from temperature-dependent Shubnikov-de Haas oscillations. The effective masses, in the unit of the free-electron mass, strongly depend on the width of the quantum wells and are 0.14-0.16 for the spin-degenerated subbands in an 8-nm-thick quantum well and 0.44-0.52 for one of the spin-split subbands in a 25-nm-thick quantum well. Furthermore, by fitting the weak antilocalization correction to the classical magnetoresistance at low magnetic fields, we obtained the phase coherence length of the system. The phase coherence length increases with hole density, reaching maxima of around 1100 and $600 \mathrm{~nm}$ for the 8and 25-nm-thick quantum wells, respectively. These achievements build upon our previous results on GaSb quantum wells and further our understanding of their properties. They therefore lay the groundwork for realizing spin-based electronics based on the strong spin-orbit interaction in this promising system.
\end{abstract}

DOI: 10.1103/PhysRevResearch.2.033383

\section{INTRODUCTION}

$\mathrm{GaSb}$ is a III-V compound semiconductor with a strong spin-orbit interaction. Recently, GaSb has been attracting attention as half of the predicted quantum spin Hall insulator InAs/GaSb double quantum well (QW), in which a 7- to 8-nm-thick GaSb QW is used to form a hybridization gap with the neighboring InAs QW [1-7]. In addition, the hole transport in $\mathrm{GaSb}$ is of technological importance for highperformance $p$-type field-effect transistors (FETs) utilizing high hole mobility at room temperature [8-20] and also for realizing spin-based electronics utilizing the aforementioned strong spin-orbit interaction [21]. However, compared with p-type GaAs [22-27] and SiGe QWs [28-31], the hole transport in GaSb QWs has been much less studied at cryogenic temperatures and considerable research has to be carried out before devices are ready for concrete applications such as hole spin qubits using quantum dots in GaSb QWs, in which long coherence times owing to the weak hyperfine interaction and fast spin control enabled by the spin-orbit interaction are

\footnotetext{
*kshibata@tohtech.ac.jp

Published by the American Physical Society under the terms of the Creative Commons Attribution 4.0 International license. Further distribution of this work must maintain attribution to the author(s) and the published article's title, journal citation, and DOI.
}

expected [32-35]. We have recently reported hole transport in a heavily doped 20-nm-thick GaSb QW and characterized the hole effective masses and phase coherence lengths [36]. We also induced holes electrically in an undoped GaSb QW and observed the integer quantum Hall effect [37]. In this study, we have gone a step further and characterized the effective mass $m^{*}$ and phase coherence length $l_{\phi}$ for such electrically induced two-dimensional (2D) hole systems in 8- and 25-nmthick undoped GaSb QWs.

\section{EXPERIMENTAL METHODS}

Undoped $\mathrm{GaSb} / \mathrm{AlSb}$ heterostructures were grown in the [100] direction on undoped GaSb substrates by molecular beam epitaxy. We measured samples with 8- and 25-nm-thick GaSb channels sandwiched between AlSb barrier layers. The thickness of the upper AlSb barrier layer was $20 \mathrm{~nm}$, while that of the lower AlSb barrier layer was $100 \mathrm{~nm}$. We formed recessed ohmic contacts to contact the electrically induced $2 \mathrm{D}$ hole systems [37-41]. The samples were patterned into large Hall bars (channel width of $100 \mu \mathrm{m}$ and length of $450 \mu \mathrm{m}$ ) and the transport channel was defined by top-gate electrodes. The sample fabrication method is described in detail elsewhere [37]. The magnetotransport properties of the samples were measured in the dark in both a variable-temperature insert (base temperature $1.5 \mathrm{~K}$ ) and a dilution refrigerator (base temperature $20 \mathrm{mK}$ ) using a standard low-frequency $(13 \mathrm{~Hz})$ lock-in technique. The magnetic field $B$ was applied 

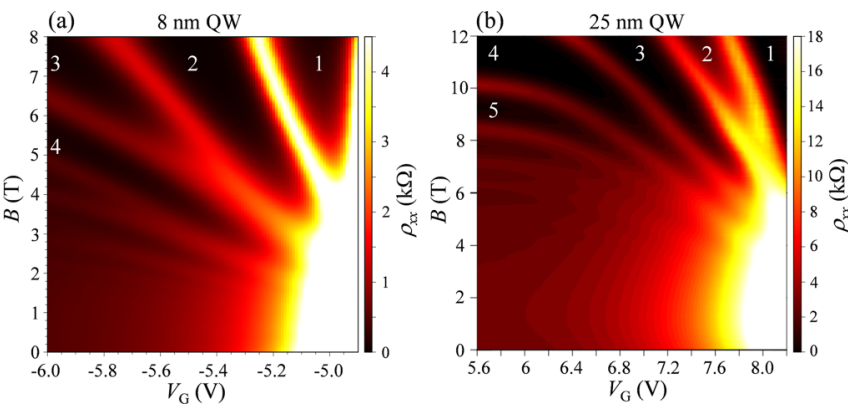

FIG. 1. (a) Landau fan diagram of longitudinal resistivity $\rho_{x x}$ for 8-nm-thick QW measured at $1.5 \mathrm{~K}$. (b) Landau fan diagram of $\rho_{x x}$ for 25-nm-thick QW measured at $100 \mathrm{mK}$. The numbers indicate the corresponding filling factors of the quantum Hall states.

perpendicular to the sample surface. In the absence of charge carriers in the QW, the resistance of the samples is typically $\sim 10 \mathrm{M} \Omega$, meaning that the samples are insulating [37].

\section{RESULTS}

Figures 1 (a) and 1(b) show the longitudinal resistivity $\rho_{x x}$ for the 8- and 25-nm-thick QWs, respectively, as a function of magnetic field and gate voltage. Dark regions with vanishing $\rho_{x x}$ indicate quantum Hall phases with integer filling factors. At a low gate voltage, clear Shubnikov-de Haas $(\mathrm{SdH})$ oscillations are observed in $\rho_{x x}$ at a low magnetic field. The SdH oscillations start to be visible at $B \sim 1 \mathrm{~T}$ $\left(V_{\mathrm{G}}=-6 \mathrm{~V}\right)$ and $B \sim 2 \mathrm{~T}\left(V_{\mathrm{G}}=5.6 \mathrm{~V}\right)$ in the 8- and 25 nm-thick QWs, respectively [see Figs. 2(a) and 3(a)]. In both samples, at a low magnetic field, the Hall resistivity $\rho_{x y}$ linearly increases with increasing $B$ (not shown), as expected from the Drude model of single-carrier transport. The Hall density and mobility derived from $\rho_{x x}$ and $\rho_{x y}$ increase with decreasing $V_{\mathrm{G}}$, indicating hole transport. The Hall mobility in the 8-nm-thick QW [maximum $20000 \mathrm{~cm}^{2} \mathrm{~V}^{-1} \mathrm{~s}^{-1}$ at $5.9 \times$ $\left.10^{11} \mathrm{~cm}^{-2}\left(V_{\mathrm{G}}=-6 \mathrm{~V}\right)\right]$ is higher than that in the $25-\mathrm{nm}-$ thick QW [maximum $10000 \mathrm{~cm}^{2} \mathrm{~V}^{-1} \mathrm{~s}^{-1}$ at $1 \times 10^{12} \mathrm{~cm}^{-2}$ $\left.\left(V_{\mathrm{G}}=5.6 \mathrm{~V}\right)\right]$, which results in the clearer $\mathrm{SdH}$ oscillations in the 8-nm-thick QW at a low magnetic field. Note that the Landau fan for the 25-nm-thick QW does not appear to be straight, which reflects the saturation of the carrier density at a low $V_{\mathrm{G}}$ owing to carrier accumulation in the capping $\mathrm{GaSb}$ layer as reported previously [37]. The very different gate voltage ranges between the 8- and 25-nm-thick QWs originate from the difference in the thickness of the capping layer (the thickness of the capping layer is $\sim 1 \mathrm{~nm}$ for the 8-nm-thick QW, while that for the 25 -nm-thick QW is $3 \mathrm{~nm}$ ) and the trapping of charges in it (the effect of the capping layer on the transport properties in undoped $\mathrm{GaSb}$ QWs is discussed in detail in Ref. [37] and its supplemental material). The temperature dependence of the $\mathrm{SdH}$ oscillations allows us to determine $m^{*}$ and quantum scattering time $\tau_{\mathrm{q}}$, as described in the following.

We are first going to determine $m^{*}$ in the 8-nm-thick QW. Figure 2(a) shows $\rho_{x x}$ as a function of $B$ at different $V_{\mathrm{G}}$ values measured for the 8-nm-thick QW exhibiting $\mathrm{SdH}$ oscillations. The corresponding power spectra calculated by (a)

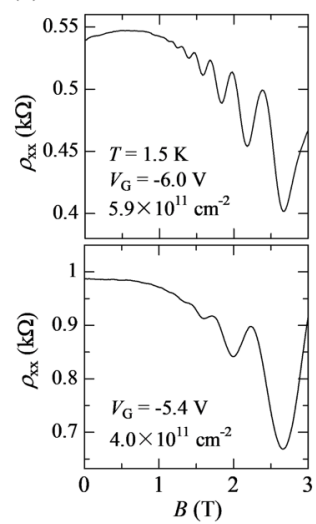

(d)

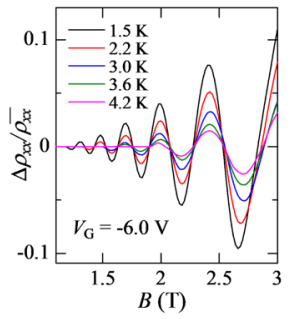

(e)

(b)

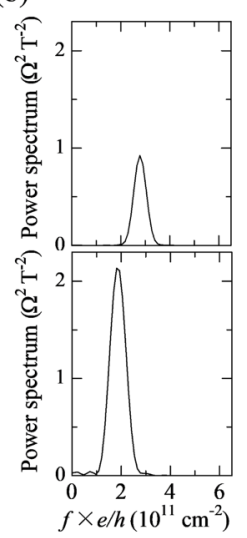

(c)
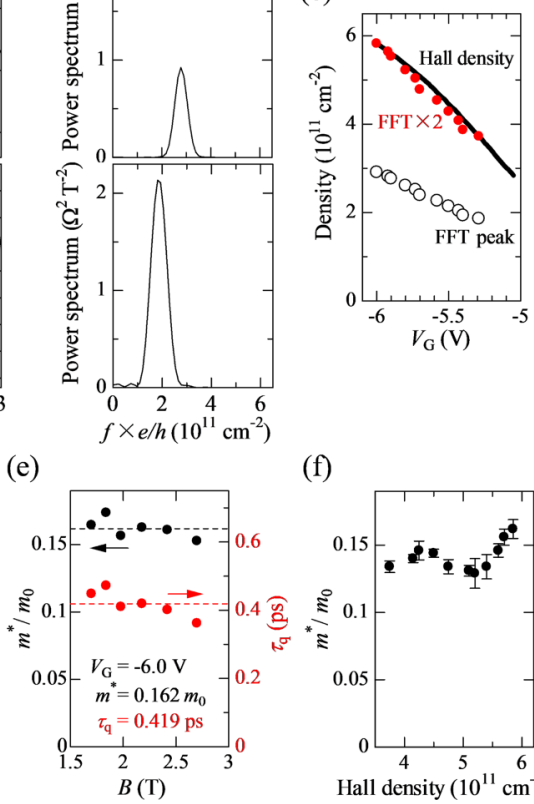

(f)

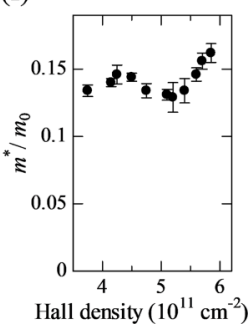

FIG. 2. (a) Examples of SdH oscillations in $\rho_{x x}$ at different $V_{\mathrm{G}}$ values for 8-nm-thick QW. (b) Power spectra obtained by Fourier transforming the $\rho_{x x}$ versus $1 / B$ traces from (a). (c) Density as a function of $V_{\mathrm{G}}$. The solid line shows the Hall density derived from the transverse resistivity at a low magnetic field. Open circles are plotted using the peak position in (b). Red circles are plotted at twice the FFT peak positions. (d) Temperature dependence of relative SdH oscillation amplitude $\Delta \rho_{x x} / \bar{\rho}_{x x}$ at $V_{\mathrm{G}}=-6 \mathrm{~V}$ after subtracting slowly varying background $\Delta \rho_{x x}\left(\Delta \rho_{x x}=\rho_{x x}-\bar{\rho}_{x x}\right)$. (e) Effective mass $m^{*}$ and quantum scattering time $\tau_{\mathrm{q}}$ up to $B=3 \mathrm{~T}$ found from (d) using Eq. (1) for many minima and maxima. The dashed lines mark the averages of $m^{*}$ and $\tau_{\mathrm{q}}$. (f) Effective mass as a function of Hall density.

the fast Fourier transform (FFT) of $\rho_{x x}$ versus $1 / B$ are displayed in Fig. 2(b), showing the evolution of the spectrum on changing $V_{\mathrm{G}}$. The frequency axis $f$ is converted to a density axis by multiplication by $e / h$ without making any assumptions regarding spin degeneracy. A single peak is observed in the entire $V_{\mathrm{G}}$ range for the 8-nm-thick QW, indicating single-band transport. Figure 2(c) shows the density as a function of $V_{\mathrm{G}}$. The solid line is the Hall density derived from $\rho_{x y}$. The FFT peak position at each $V_{\mathrm{G}}$ is plotted in Fig. 2(c) as open circles. The FFT peak is located at almost half of the Hall density, indicating that spin states are degenerate in this magnetic field/density range.

In order to determine $m^{*}$, we measured the temperature dependence of the $\mathrm{SdH}$ oscillations at each $V_{\mathrm{G}}$. In the 8-nmthick QW, the oscillation amplitude slowly decreases with increasing temperature. Therefore, the $\mathrm{SdH}$ oscillations were measured using the variable-temperature insert in a wide temperature range of $1.5 \mathrm{~K} \leqslant T \leqslant 4.2 \mathrm{~K}$. For example, Fig. 2(d) shows the temperature dependence of the relative $\mathrm{SdH}$ oscillation amplitude $\Delta \rho_{x x} / \bar{\rho}_{x x}$ at $V_{\mathrm{G}}=-6 \mathrm{~V}$ after subtracting the slowly varying background $\bar{\rho}_{x x}$. We employ the well-known 
(a)

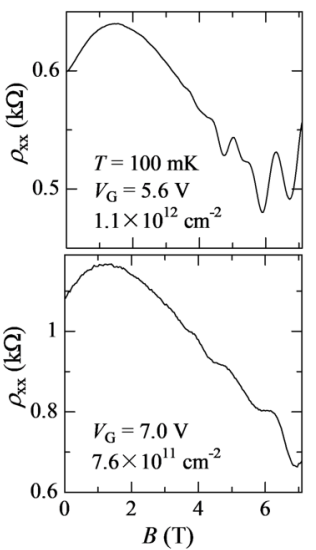

(b)

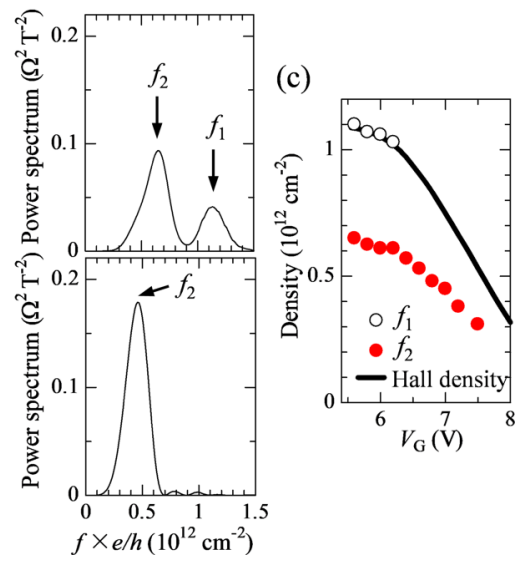

FIG. 3. (a) Examples of $\mathrm{SdH}$ oscillations in $\rho_{x x}$ at different $V_{\mathrm{G}}$ values for 25-nm-thick QW. (b) Power spectra obtained by FFT of $\rho_{x x}$ versus $1 / B$ traces from (a). (c) Carrier density as a function of $V_{\mathrm{G}}$. The solid line shows the Hall density derived from the transverse resistivity at a low magnetic field. Open circles are plotted using the peak position $f_{1}$ in (b). The red circles are plotted using the peak position $f_{2}$ in (b).

expression describing the oscillation amplitude $\Delta \rho_{x x}$,

$$
\frac{\Delta \rho_{x x}}{\bar{\rho}_{x x}}=4 \exp \left(-\frac{\pi}{\omega_{\mathrm{c}} \tau_{\mathrm{q}}}\right) \frac{2 \pi^{2} k_{\mathrm{B}} T / \hbar \omega_{\mathrm{c}}}{\sinh 2 \pi^{2} k_{\mathrm{B}} T / \hbar \omega_{\mathrm{c}}},
$$

to fit the data, where $\bar{\rho}_{x x}$ is the background magnetoresistance, $\omega_{\mathrm{c}}=e B / m^{*}$ the cyclotron frequency, and $T$ the temperature [42]. Equation (1) is valid for $\Delta \rho_{x x} / \bar{\rho}_{x x} \ll 1$. Fitting the decay of the oscillation amplitude with the temperature at different minima and maxima using Eq. (1) gives Fig. 2(e). We see that $m^{*}$ and $\tau_{\mathrm{q}}$ are independent of $B$ and obtain $m^{*} / m_{0}=$ $0.162 \pm 0.007$ and $\tau_{\mathrm{q}}=(0.419 \pm 0.004) \mathrm{ps}$, where $m_{0}$ is the free-electron mass. Figure 2(f) shows $m^{*}$ as a function of Hall density for the 8-nm-thick QW. The effective mass is almost independent of the Hall density and is $0.14-0.16$ in units of the free-electron mass, suggesting an almost parabolic band structure in this density range. The quantum scattering time was found to be $\tau_{\mathrm{q}}=0.3-0.4 \mathrm{ps}$ in the observed $V_{\mathrm{G}}$ range. A similar hole effective mass of $m^{*}=0.136 m_{0}$ was reported for a $\mathrm{GaSb}(7 \mathrm{~nm}) / \mathrm{InAs}(11 \mathrm{~nm})$ double-QW structure operated in the hole regime [43], suggesting that the hybridization between GaSb and InAs QWs does not affect the hole effective mass deep in the GaSb valence band, away from the charge neutrality point.

Next, we perform a similar analysis to determine $m^{*}$ in the 25-nm-thick QW. The situation is different for the 25-nmthick QW. Figure 3(a) shows SdH oscillations at different $V_{\mathrm{G}}$ values measured for the 25 -nm-thick $\mathrm{QW}$. The corresponding power spectra calculated by the FFT of $\rho_{x x}$ versus $1 / B$ are displayed in Fig. 3(b). The frequency axis $f$ is again converted to a density axis by multiplication by $e / h$. In this case, two frequencies, $f_{1}$ and $f_{2}$, are visible in the spectrum at $V_{\mathrm{G}}=$ $5.6 \mathrm{~V}$. The $f_{1}$ peak gradually disappears and its frequency decreases with increasing $V_{\mathrm{G}}$, while the $f_{2}$ peak is observed up to a higher $V_{\mathrm{G}}$ of $\sim 7.5 \mathrm{~V}$. Figure 3(c) shows the density as a function of $V_{\mathrm{G}}$ for the 25 -nm-thick QW. The solid line is the Hall density derived from $\rho_{x y}$. The FFT peak positions

(a)

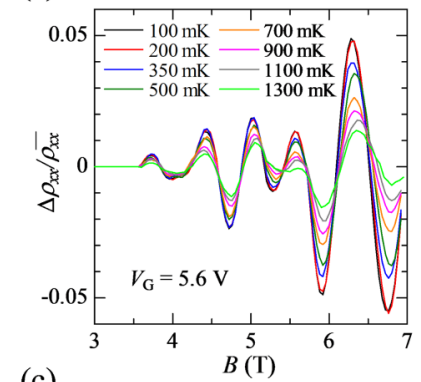

(c)

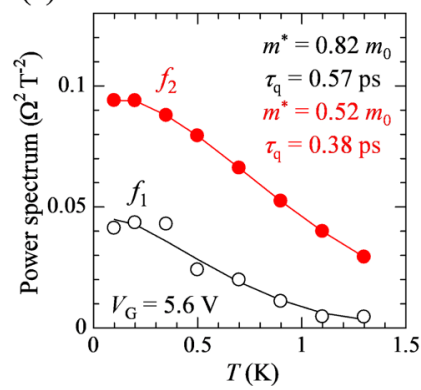

(b)

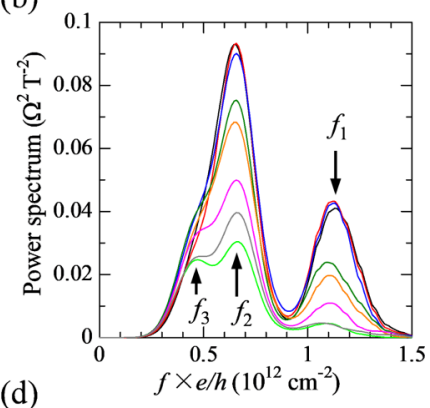

(d)

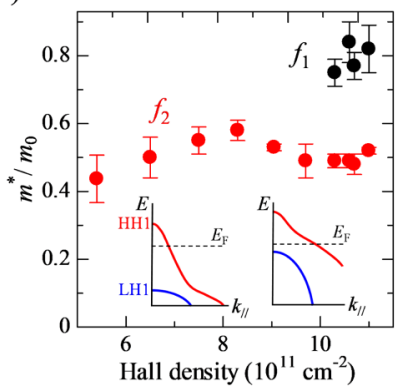

FIG. 4. (a) Temperature dependence of relative $\mathrm{SdH}$ oscillation amplitude $\Delta \rho_{x x} / \bar{\rho}_{x x}$ at $V_{\mathrm{G}}=5.6 \mathrm{~V}$ for 25-nm-thick QW. (b) Decay of the power spectrum with increasing temperature at $V_{\mathrm{G}}=5.6 \mathrm{~V}$. (c) FFT peak heights at frequencies $f_{1}$ (black) and $f_{2}$ (red) versus temperature, together with their fits (solid lines). (d) Effective mass as a function of Hall density. The insets shows the schematic valence band structures for (left) 8-nm-thick and (right) 25-nm-thick QWs. The dashed line shows the position of the Fermi level $E_{\mathrm{F}}$.

for the $f_{1}$ and $f_{2}$ peaks are plotted in Fig. 3(c) as open and red circles, respectively. We see that the $f_{1}$ peak matches the Hall density, indicating that the $f_{1}$ peak corresponds to the total density. The $f_{2}$ peak is located at $\sim 0.6 \times$ (total density), suggesting lifted spin degeneracy and another missing peak at $\sim 0.4 \times$ (total density).

Figure 4(a) shows the temperature dependence of the relative $\mathrm{SdH}$ oscillation amplitude $\Delta \rho_{x x} / \bar{\rho}_{x x}$ at $V_{\mathrm{G}}=5.6 \mathrm{~V}$ after subtracting the slowly varying background $\bar{\rho}_{x x}$ for the $25-\mathrm{nm}$ thick QW. Here, an intricate analysis is required because two frequencies contribute to the $\mathrm{SdH}$ oscillations. We applied a method to analyze the data [22,36], that considers the power spectra of the SdH oscillations. We fitted the heights of the peaks corresponding to the different subbands as a function of temperature to the peak heights in the power spectra of simulated data sets produced using the Fourier transform of Eq. (1). Modifications such as the choice of the magnetic field range and windowing were applied to both real and simulated data sets, ensuring consistency. The fitting parameters were again $m^{*}$ and $\tau_{\mathrm{q}}$. Figure 4 (b) shows the decay of the power spectrum with increasing temperature at $V_{\mathrm{G}}=5.6 \mathrm{~V}$. With increasing temperature, the height of the $f_{2}$ peak is suppressed and, correspondingly, the missing peak $f_{3}$ becomes visible. We confirmed that $f_{2}+f_{3}$ corresponds to $f_{1}$. In Fig. 4(c), we show the peak heights at the frequencies $f_{1}$ and $f_{2}$ versus temperature, together with their fits (solid lines). For the total density, $f_{1}$, we obtained $m^{*} / m_{0}=0.82 \pm 0.07$ and $\tau_{\mathrm{q}}=(0.57 \pm 0.05) \mathrm{ps}$, and for the $f_{2}$ subband, we obtained $m^{*} / m_{0}=0.52 \pm 0.01$ and $\tau_{\mathrm{q}}=(0.38 \pm 0.01)$ ps. From these 
results, the effective mass for the missing peak $f_{3}$, which is observed only at high temperatures in Fig. 4(b), was empirically estimated to be $m^{*} / m_{0}=0.82-0.52=0.3$ [22] .

Figure 4(d) shows the effective mass $m^{*} / m_{0}$ as a function of Hall density, plotted for the $f_{1}$ (total density) and the $f_{2}$ subband. The effective mass for the $f_{2}$ subband does not strongly depend on the Hall density, again suggesting an almost parabolic band structure in this density regime. If we compare $m^{*}$ between 8 - and 25 -nm-thick QWs at the closest density, the value of $m^{*} / m_{0}=0.44 \pm 0.07$ (at $5.3 \times 10^{11} \mathrm{~cm}^{-2}$ ) for the $f_{2}$ subband of the 25 -nm-thick QW is much higher than $m^{*} / m_{0}=0.134 \pm 0.009$ (at $5.4 \times$ $10^{11} \mathrm{~cm}^{-2}$ ) for the 8-nm-thick QW. The well width dependence of $m^{*}$ can be understood qualitatively from the complex shape of the two-dimensional (2D) valence bands $[36,44,45]$. As illustrated in the insets of Fig. 4(d), in the 8-nm-thick QW, owing to the strong quantum confinement, the heavy hole (HH)-light hole ( $\mathrm{LH})$ band splitting is large and the Fermi energy is much higher than the HH-LH anticrossing, leading to the small $m^{*}$ for the 8-nm-thick QW. On the other hand, for the thicker QW, the HH-LH band splitting is small and the Fermi level is closer to the HH-LH anticrossing, leading to the larger $m^{*}$ for the 25-nm-thick QW.

It is worthwhile to compare $m^{*}$ in the 25 -nm-thick QW with the recently reported results in a 20-nm-thick GaSb QW at the same total density, although the QW width is slightly different. In a heavily doped 20-nm-thick GaSb QW, two spin-split subbands were observed and $m^{*}$ of one subband $m^{*} / m_{0}=0.770 \pm 0.004$ was reported $\left(m^{*}\right.$ of the other subband could not be resolved owing to too small density) at the lowest total density of $1.0 \times 10^{12} \mathrm{~cm}^{-2}$ [36]. In this work, $m^{*}$ in the undoped 25-nm-thick GaSb QW at the same total density is $m^{*} / m_{0}=0.49 \pm 0.02$ for the $f_{2}$ subband. The self-consistent $\boldsymbol{k} \cdot \boldsymbol{p}$ band structure calculations have been performed for a 20-nm-thick GaSb QW and the effective masses of the spin-split ground and excited state subbands were obtained $[36,46]$. According to these calculations, a significant zero-field spin splitting of the ground subband owing to the spin-orbit interaction is observed in the entire density range. Although it is known that the calculated $m^{*}$ shows differences from the experimental value in $2 \mathrm{D}$ hole systems owing to the complicated structure of the valence band $[22,36,46]$, the calculated $m^{*}$ were $m^{*} / m_{0} \sim 0.5$ for both spin-split subbands of the 20-nm-thick QW at $1.0 \times 10^{12} \mathrm{~cm}^{-2}$. This value is consistent with our experimental result and matches it more closely than the experimentally obtained result in the $20-\mathrm{nm}$ thick QW described above.

In the presence of strong spin-orbit interaction, negative magnetoconductivity is observed at a low magnetic field owing to the weak antilocalization (WAL) [47]. By analyzing the WAL correction to the classical magnetoresistance, we obtain the phase coherence length $l_{\phi}$ and the spin-orbit length $l_{\mathrm{so}}$ of the system. To investigate the WAL, we measure the magnetic field dependence of $\rho_{x x}$ and $\rho_{x y}$ for a fixed $V_{\mathrm{G}}$ and calculate the longitudinal conductivity $\sigma_{x x}$ by inverting $\rho_{x x}$ and $\rho_{x y}$. Then, we subtract the classical Drude conductivity from the total conductivity to extract the WAL conductivity correction. For the 8-nm-thick QW, we observe only a single FFT peak at half of the Hall density [see Figs. 2(b) and 2(c)], indicating single-band transport. Thus, we fit $\sigma_{x x}$ with the (a)

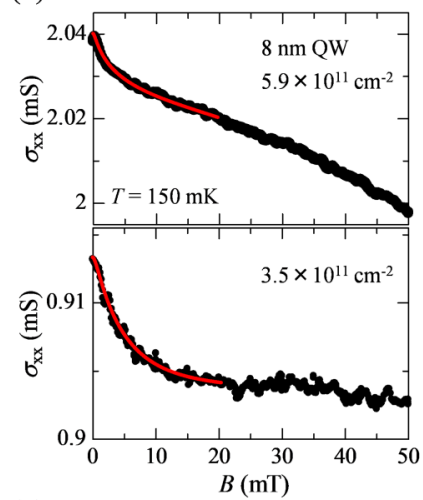

(c)

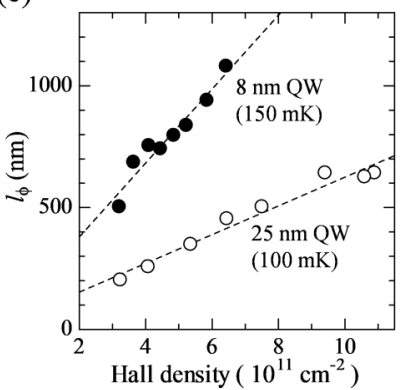

(b)

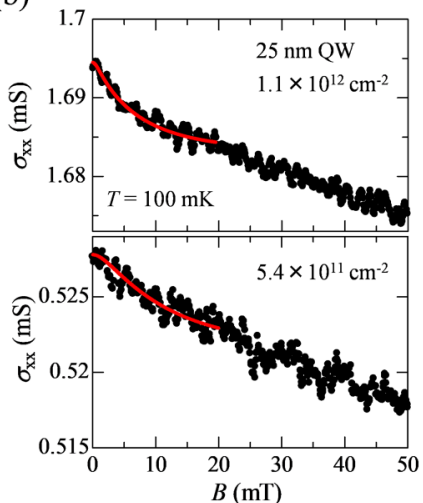

(d)

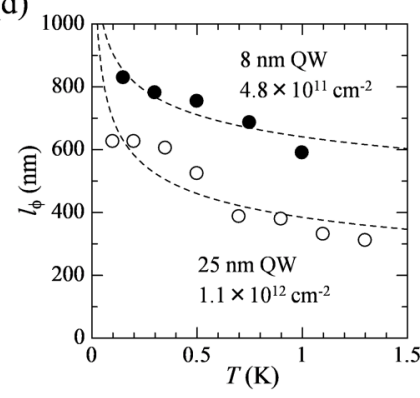

FIG. 5. Examples of WAL correction to longitudinal conductivity $\sigma_{x x}$ at different hole densities plotted for (a) 8-nm-thick and (b) 25-nm-thick QWs, together with their fits (red line) using Eq. (2) in the range $|B|<20 \mathrm{mT}$. (c) Phase coherence length $l_{\phi}$ found from WAL correction as a function of Hall density for 8-nm-thick (closed circles) and 25-nm-thick (open circles) QWs. The dashed lines are guides for the eyes. (d) Temperature dependence of $l_{\phi}$ for 8-nm-thick $\left(4.8 \times 10^{11} \mathrm{~cm}^{-2}\right)$ and 25-nm-thick $\left(1.1 \times 10^{12} \mathrm{~cm}^{-2}\right)$ QWs. Dashed lines are fits with the power law dependence $T^{\gamma}$.

classical Drude conductivity in the 8-nm-thick QW. For the 25-nm-thick QW, two-band transport is observed as discussed earlier. Therefore, we fit $\sigma_{x x}$ with a two-band model of the form $\sigma_{x x}=\sigma_{x x, 2}+\sigma_{x x, 3}$, where $\sigma_{x x, 2}\left(\sigma_{x x, 3}\right)$ is the conductivity of the band with a density of $f_{2} \times e / h\left(f_{3} \times e / h\right)$ in Fig. 3 . We neglect intersubband scattering and fix the densities to $f_{1} \times e / h$ and $f_{2} \times e / h$, resulting in two fitting parameters, $\mu_{2}$ and $\mu_{3}$, which are the mobilities of the subbands. Both oneband and two-band models give the parabolic background, explaining the observed positive magnetoresistance around $B=0$ [see, for example, Fig. 3(a)]. Note that $\rho_{x y}$ is essentially linear in $B$ despite the presence of two hole species for the 25-nm-thick QW. After subtracting the parabolic background from $\sigma_{x x}$ and obtaining $\delta \sigma_{x x}$, we shift the traces such that $\delta \sigma_{x x}(0)=0$ and fit them with

$\delta \sigma_{x x}=\frac{e^{2}}{\pi h}\left[g\left(\frac{B_{\phi}+B_{\text {so }}}{B}\right)+\frac{1}{2} g\left(\frac{B_{\phi}+2 B_{\text {so }}}{B}\right)-\frac{1}{2} g\left(\frac{B_{\phi}}{B}\right)\right]$,

where $g(x)=\Psi(1 / 2+x)-\ln x$ and $\Psi$ is the digamma function [48]. The quantities $B_{\phi}=h / 4 e l_{\phi}^{2}$ and $B_{\mathrm{so}}=h / 4 e l_{\mathrm{so}}^{2}$ are the fitting parameters. We are interested in $l_{\phi}$ and $l_{\text {so }}$. Figures 5(a) and 5(b) show several typical fits at different Hall densities for the 8- and 25-nm-thick QWs, respectively. The fitting range is $|B|<20 \mathrm{mT}$. We find that $l_{\phi}$ is mostly 
independent of the fitting range, whereas $l_{\mathrm{so}}$ is markedly different for different fitting ranges. This occurs because $l_{\phi}$ describes the well-defined peak shape of $\delta \sigma_{x x}$ around $B=0$, where the background is flat. In contrast, $l_{\text {so }}$ describes the behavior of $\delta \sigma_{x x}$ away from the peak and is therefore affected by the details of the crude background subtraction, which neglects particle-particle interactions, for instance. Thus, there is a systematic error in $l_{\mathrm{so}}[36,49]$.

Figure 5(c) presents the extracted $l_{\phi}$ versus Hall density. $l_{\phi}$ increases with the density, reaching maxima of around 1100 $\mathrm{nm}$ (8-nm-thick QW) and $600 \mathrm{~nm}$ (25-nm-thick QW). We do not plot $l_{\mathrm{so}}$ in light of the discussion outlined above but mention here that $l_{\text {so }}$ does not seem to depend on the Hall density and is around 100-200 nm in both QWs. If we compare $l_{\phi}$ in the doped and undoped structures despite the small difference in the QW width, $l_{\phi}$ in the undoped 25-nm-thick QW is $l_{\phi} \sim$ $600 \mathrm{~nm}$ at a density of $\sim 10^{12} \mathrm{~cm}^{-2}$ at $100 \mathrm{mK}$, which is larger than $l_{\phi} \sim 400 \mathrm{~nm}$ at the same density and temperature in the heavily doped 20-nm-thick QW [36]. The reason for the larger $l_{\phi}$ in the undoped QW might be the reduced scattering by the ionized impurities in it. Increasing the temperature quenches the WAL correction. Figure 5(d) illustrates the decrease in $l_{\phi}$ at two Hall densities in the different samples upon increasing the temperature. It is known that the scattering mechanism of carriers can be discussed from the temperature dependence of $l_{\phi}$. We fitted the data points with a function of the form $A T^{\gamma}$ and obtained $\gamma \sim-0.15 \pm 0.03$ and $\gamma \sim-0.26 \pm 0.05$ for the 8- and 25-nm-thick QWs, respectively. These values are much smaller than $\gamma \sim-0.5$ expected in the case of electronelectron scattering as the dominant decoherence mechanism $[50,51]$ but closer to the results for a heavily doped $\mathrm{GaSb} \mathrm{QW}$
[36]. Currently, we cannot explain the unexpected value of the exponent $\gamma$.

\section{SUMMARY}

In summary, we have investigated the magnetotransport of electrically induced 2D hole gases in undoped GaSb/AlSb QWs. The effective masses of valence bands were evaluated from temperature-dependence measurements of $\mathrm{SdH}$ oscillations. The effective masses strongly depend on the width of the QWs and are $m^{*} / m_{0}=0.14-0.16$ for the spin-degenerated subbands in the 8-nm-thick QW and $m^{*} / m_{0}=0.44-0.52$ for one of the spin-split subbands in the 25-nm-thick QW. Phase coherence lengths derived from a weak antilocalization correction increase with hole density, reaching maxima of around 1100 and $600 \mathrm{~nm}$ for the 8and 25-nm-thick QWs, respectively. These achievements build upon our previous results on GaSb QWs and further our understanding of their properties. They therefore lay the groundwork for realizing spin-based electronics based on the strong spin-orbit interaction in this promising system.

\section{ACKNOWLEDGMENTS}

The authors acknowledge the support of the ETH FIRST Laboratory and the financial support of the Swiss Science Foundation (Schweizerischer Nationalfonds, NCCR QSIT). K.S. thanks M. Hug for his support on the effective mass analysis. This work was partly supported by Grants-in-Aid from JSPS (Grants No. 15KK0215 and No. 17H02732) and the Asahi Glass Foundation.
[1] C. L. Kane and E. J. Mele, Phys. Rev. Lett. 95, 146802 (2005).

[2] C. L. Kane and E. J. Mele, Phys. Rev. Lett. 95, 226801 (2005).

[3] B. A. Bernevig and S.-C. Zhang, Phys. Rev. Lett. 96, 106802 (2006).

[4] C. Liu, T. L. Hughes, X.-L. Qi, K. Wang, and S.-C. Zhang, Phys. Rev. Lett. 100, 236601 (2008).

[5] K. Suzuki, Y. Harada, K. Onomitsu, and K. Muraki, Phys. Rev. B 87, 235311 (2013).

[6] L. Du, I. Knez, G. Sullivan, and R.-R. Du, Phys. Rev. Lett. 114, 096802 (2015).

[7] S. Mueller, A. N. Pal, M. Karalic, T. Tschirky, C. Charpentier, W. Wegscheider, K. Ensslin, and T. Ihn, Phys. Rev. B 92, 081303(R) (2015).

[8] B. R. Bennett, R. Magno, J. B. Boos, W. Kruppa, and M. G. Ancona, Solid State Electron. 49, 1875 (2005).

[9] A. Ali, H. S. Madan, A. P. Kirk, D. A. Zhao, D. A. Mourey, M. K. Hudait, R. M.Wallace, T. N. Jackson, B. R. Bennett, J. B. Boos, and S. Datta, Appl. Phys. Lett. 97, 143502 (2010).

[10] C. Merckling, X. Sun, A. Alian, G. Brammertz, V. V. Afanas'ev, T. Y. Hoffmann, M. Heyns, M. Caymax, and J. Dekoster, J. Appl. Phys. 109, 073719 (2011).

[11] A. Nainani, T. Irisawa, Z. Yuan, B. R. Bennett, J. B. Boos, Y. Nishi, and K. C. Saraswat, IEEE Trans. Electron Devices 58, 3407 (2011).
[12] B. R. Bennett, M. G. Ancona, J. B. Boos, C. B. Canedy, and S. A. Khan, J. Cryst. Growth 311, 47 (2008).

[13] V. Tokranov, P. Nagaiah, M. Yakimov, R. J. Matyi, and S. Oktyabrsky, J. Cryst. Growth 323, 35 (2011).

[14] B. R. Bennett, T. F. Chick, M. G. Ancona, and J. B. Boos, Solid State Electron. 79, 274 (2013).

[15] M. Jeppsson, K. A. Dick, H. A. Nilsson, N. Skold, J. B. Wagner, P. Caroff, and L.-E. Wernersson, J. Cryst. Growth 310, 5119 (2008).

[16] R. A. Burke, X. Weng, M.-W. Kuo, Y.-W. Song, A. M. Itsuno, T. S. Mayer, S. M. Durbin, R. J. Reeves, and J. M. Redwing, J. Electron. Mater. 39, 355 (2010).

[17] B. M. Borg and L.-E. Wernersson, Nanotechnology 24, 202001 (2013).

[18] Z.-X. Yang, N. Han, M. Fang, H. Lin, H.-Y. Cheung, S. Yip, E.-J. Wang, T. Hung, C.-Y. Wong, and J. C. Ho, Nat. Commun. 5, 5249 (2014).

[19] Z.-X. Yang, S. Yip, D. Li, N. Han, G. Dong, X. Liang, L. Shu, T. F. Hung, X. Mo, and J. C. Ho, ACS Nano 9, 9268 (2015).

[20] M. Borg, H. Schmid, J. Gooth, M. D. Rossell, D. Cutaia, M. Knoedler, N. Bologna, S. Wirths, K. E. Moselund, and H. Riel, ACS Nano 11, 2554 (2017).

[21] R. Winkler, Spin-Orbit Coupling Effects in Two-Dimensional Electron and Hole Systems (Springer, New York, 2003). 
[22] F. Nichele, A. N. Pal, R. Winkler, C. Gerl, W. Wegscheider, T. Ihn, and K. Ensslin, Phys. Rev. B 89, 081306(R) (2014).

[23] B. Grbic, R. Leturcq, K. Ensslin, D. Reuter, and A. D. Wieck, Appl. Phys. Lett. 87, 232108 (2005).

[24] O. Klochan, J. C. H. Chen, A. P. Micolich, A. R. Hamilton, K. Muraki, and Y. Hirayama, Appl. Phys. Lett. 96, 092103 (2010).

[25] Y. Komijani, T. Choi, F. Nichele, K. Ensslin, T. Ihn, D. Reuter, and A. D. Wieck, Phys. Rev. B 88, 035417 (2013).

[26] D. Q. Wang, O. Klochan, J.-T. Hung, D. Culcer, I. Farrer, D. A. Ritchie, and A. R. Hamilton, Nano Lett. 16, 7685 (2016).

[27] A. Bogan, S. A. Studenikin, M. Korkusinski, G. C. Aers, L. Gaudreau, P. Zawadzki, A. S. Sachrajda, L. A. Tracy, J. L. Reno, and T. W. Hargett, Phys. Rev. Lett. 118, 167701 (2017).

[28] H. von Känel, M. Kummer, G. Isella, E. Müller, and T. Hackbarth, Appl. Phys. Lett. 80, 2922 (2002).

[29] A. Dobbie, M. Myronov, R. J. H. Morris, A. H. A. Hassan, M. J. Prest, V. A. Shah, E. H. C. Parker, T. E. Whall, and D. R. Leadley, Appl. Phys. Lett. 101, 172108 (2012).

[30] N. W. Hendrickx, D. P. Franke, A. Sammak, M. Kouwenhoven, D. Sabbagh, L. Yeoh, R. Li, M. L. V. Tagliaferri, M. Virgilio, G. Capellini, G. Scappucci, and M. Veldhorst, Nat. Commun. 9, 2835 (2018).

[31] N. W. Hendrickx, D. P. Franke, A. Sammak, G. Scappucci, and M. Veldhorst, Nature 577, 487 (2020).

[32] D. V. Bulaev and D. Loss, Phys. Rev. Lett. 95, 076805 (2005).

[33] J. Fischer, W. A. Coish, D. V. Bulaev, and D. Loss, Phys. Rev. B 78, 155329 (2008).

[34] J. Fischer and D. Loss, Phys. Rev. Lett. 105, 266603 (2010).

[35] P. Szumniak, S. Bednarek, B. Partoens, and F. M. Peeters, Phys. Rev. Lett. 109, 107201 (2012).

[36] M. Karalic, C. Mittag, M. Hug, T. Tschirky, W. Wegscheider, K. Ensslin, T. Ihn, K. Shibata, and R. Winkler, Phys. Rev. B 99, 115435 (2019).
[37] K. Shibata, M. Karalic, C. Mittag, T. Tschirky, C. Reichl, H. Ito, K. Hashimoto, T. Tomimatsu, Y. Hirayama, W. Wegscheider, T. Ihn, and K. Ensslin, Appl. Phys. Lett. 114, 232102 (2019).

[38] R. L. Willett, L. N. Pfeiffer, and K. W. West, Appl. Phys. Lett. 89, 242107 (2006).

[39] T. M. Lu, D. R. Luhman, K. Lai, D. C. Tsui, L. N. Pfeiffer, and K. W. West, Appl. Phys. Lett. 90, 112113 (2007).

[40] S. Sarkozy, K. Das Gupta, F. Sfigakis, I. Farrer, H. Beere, R. Harrell, D. Ritchie, and G. Jones, ECS Trans. 11, 75 (2007).

[41] S. Sarkozy, K. Das Gupta, C. Siegert, A. Ghosh, M. Pepper, I. Farrer, H. E. Beere, D. A. Ritchie, and G. A. C. Jones, Appl. Phys. Lett. 94, 172105 (2009).

[42] T. Ando, A. B. Fowler, and F. Stern, Rev. Mod. Phys. 54, 437 (1982).

[43] X. Mu, G. Sullivan, and R.-R. Du, Appl. Phys. Lett. 108, 012101 (2016).

[44] J. A. Kash, M. Zachau, M. A. Tischler, and U. Ekenberg, Phys. Rev. Lett. 69, 2260 (1992).

[45] H. Zhua, K. Lai, D. C. Tsui, S. P. Bayrakci, N. P. Ong, M. Manfra, L. Pfeiffer, and K. West, Solid State Commun. 141, 510 (2007).

[46] M. Karalic, Electronic transport in hybridized electron-hole bilayers, Ph.D. thesis, ETH Zurich, 2019, https://www.researchcollection.ethz.ch/handle/20.500.11850/378337.

[47] S. Hikami, A. I. Larkin, and Y. Nagaoka, Prog. Theor. Phys. 63, 707 (1980).

[48] S. V. Iordanskii, Y. B. Lyanda-Geller, and G. E. Pikus, Zh. Eksp. Teor. Fiz. Pis'ma Red 60, 199 (1994).

[49] B. Grbić, R. Leturcq, T. Ihn, K. Ensslin, D. Reuter, and A. D. Wieck, Phys. Rev. B 77, 125312 (2008).

[50] T. Ihn, Semiconductor Nanostructures: Quantum States and Electronic Transport (Oxford University Press, Oxford, 2009).

[51] S. Chakravarty and A. Schmid, Phys. Rep. 140, 193 (1986). 